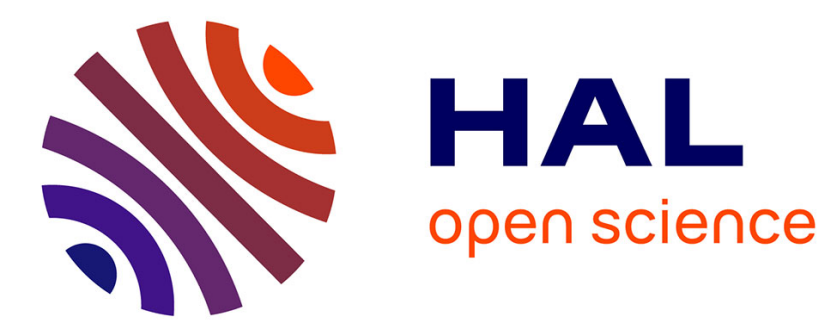

\title{
Using computer algebra to diagonalize some Kane matrices
}

\author{
Claude-Pierre Jeannerod, Nicolas Maillard
}

\section{To cite this version:}

Claude-Pierre Jeannerod, Nicolas Maillard. Using computer algebra to diagonalize some Kane matrices. Journal of Physics A: Mathematical and General (1975 - 2006), 2000, 33 (14), pp.2857-2870. 10.1088/0305-4470/33/14/316 . hal-03420701

\section{HAL Id: hal-03420701 \\ https://hal.inria.fr/hal-03420701}

Submitted on 9 Nov 2021

HAL is a multi-disciplinary open access archive for the deposit and dissemination of scientific research documents, whether they are published or not. The documents may come from teaching and research institutions in France or abroad, or from public or private research centers.
L'archive ouverte pluridisciplinaire HAL, est destinée au dépôt et à la diffusion de documents scientifiques de niveau recherche, publiés ou non, émanant des établissements d'enseignement et de recherche français ou étrangers, des laboratoires publics ou privés. 


\title{
Using computer algebra to diagonalize some Kane matrices
}

\author{
C-P Jeannerod $\dagger, \mathbf{N}$ Maillard $\dagger$ t \\ $\dagger$ LMC-IMAG, Grenoble, France \\ E-mail: Claude-Pierre.Jeannerod@imag.fr \\ † Observatoire de Grenoble, Grenoble, France \\ E-mail: Nicolas.Maillard@imag.fr
}

\begin{abstract}
In semi-conductor theory, applying the $k p$-method to the monodimensional Schrödinger equation leads to a symmetric perturbed eigenvalue problem [8], i.e. to the diagonalization of a matrix $A(\varepsilon)$ depending on a small parameter $\varepsilon$, symmetric $\forall \varepsilon \in \mathbb{R}$. The eigenelements of $A(\varepsilon)$ admit expansions in fractional powers of $\varepsilon$ (Puiseux series). Usually, physicists solve this problem by using Schrödinger perturbation formulas under some restrictive conditions, which make perturbed eigenvector symbolic approximation impossible. This is illustrated by the modified Kane matrix [5]. To solve this problem completely from a symbolic computing point of view, we consider the symmetric perturbed eigenvalue problem in the case of analytic perturbations $[2,9]$. We first review the classical characteristic polynomial approach, showing why it may be not optimal. We also present a direct matricial algorithm [7]: transforming the analytic matrix $A(\varepsilon)$ into its so-called $q$-reduced form allows to recover the information we need for the eigenvalues. This alternative method, as well as the classical one, can be described in terms of the Newton polygon. However, our approach uses only a finite number of terms of $A(\varepsilon)$ and is more suitable for large matrices and a low approximation order. Besides, we show that the $q$-reduction process can simultaneously provide symbolic approximations of both the perturbed eigenvalues and eigenvectors. The implementation of this algorithm in MAPLE is used to diagonalize the modified Kane matrix up to a given order.
\end{abstract}

Submitted to: J. Phys. A: Math. Gen. 


\section{Introduction}

Eigenvalue problems arise naturally in many physical and mathematical applications. One of the physical fields where they are most encountered is quantum mechanics. When addressing the question of determining the stationary states of an electron-like particle in a quantum well of semi-conductors ( $\mathrm{SC}$ 's), one has to solve a Schrödinger equation, i.e. to compute the eigenelements of an Hamiltonian operator $\mathcal{H}_{0}$. The Schrödinger equation studied therein will be supposed to be monodimensional and stationary. The crystalline structure of SC's is periodic and the wavefunctions and energies of the particle depend on a real parameter $k$. The Hamiltonian $\mathcal{H}_{k}$ can be written as the sum of $\mathcal{H}_{0}$ and of a perturbative part, which is polynomial in $k$. The $k p$-perturbation theory [8] leads to a matricial representation of operator $\mathcal{H}_{k}$. Projecting operator $\mathcal{H}_{k}$ on a finite dimension basis yields a quadratic polynomial matrix $H(k)=H_{0}+H_{1} k+H_{2} k^{2}$, which is symmetric for all $k$ real: $H^{*}(k)=H(k), \forall k \in \mathbb{R}$. We are therefore led to the diagonalization of $H(k)$, that is, assuming that $k$ is in some neighborhood of zero, to the resolution of a symmetric perturbed eigenvalue problem [9, p.120]. It mainly consists in determining the first terms of the $k$-expansions of both the perturbed eigenvalues and eigenvectors of $H(k)$.

The traditional way physicists solve this problem is based on the Schrödinger perturbation theory ([3], [2, p.102]) which approximates these $k$-expansions up to any order $q \in \mathbb{N}$ under some restrictive conditions on the relative size of the matrix elements [10]. The Schrödinger formulas have been used to approximate up to order $q=2$ the perturbed eigenvalues of a particular $H(k)$ matrix, known as the modified Kane matrix [5]. Yet, Schrödinger conditions imply a simplification of the initial problem $\mathcal{H}_{k}$, which does not allow to derive truncated $k$-expansions for the corresponding perturbed eigenvectors. Thus, to approximate both the perturbed eigenvalues and eigenvectors of the matrix $H(k)$ corresponding to the actual operator $\mathcal{H}_{k}$, a more general approach is needed.

The problem of the change of the eigenelements of a matrix under small perturbations has been widely studied $[2,9,16]$. In general, the perturbations considered are not polynomial but analytic perturbations. Besides, the small parameter $k$ being an indeterminate, computer algebra is particularly suitable for an algorithmic approach. The central tools we will use are the so-called Newton polygon of an analytic matrix [2, p.411], together with its associated Newton polynomials. Their computation gives for each perturbed eigenvalue the first-order term of its $k$-expansion. Having determined this first-order term, one may recursively get the following terms up to any order. Then, in the symmetric case, the corresponding perturbed eigenvector can be approximated up to any order by using some recurrence formulas.

To determine both the Newton polygon and its associated Newton polynomials, the classical method consists in computing the characteristic polynomial $\chi(k, \lambda)=$ $\operatorname{det}(\lambda I-H(k))$ of $H(k)$ and solving the algebraic equation $\chi(k, \lambda)=0$. However, the computation of the characteristic polynomial of a matrix depending on a parameter 
may become dramatically time-consuming when the dimension of the matrix and/or the degree of the perturbation increase(s) (see [1] for a discussion). Moreover, although there exists an efficient algorithm to solve $\chi(k, \lambda)=0$ in the case of polynomial perturbations [15], analytic perturbations can not be dealt with.

Another method is proposed, which aims at simultaneously approximating both the perturbed eigenvalues and eigenvectors of a symmetric analytic matrix $H(k)$, without computing the characteristic polynomial of $H(k)$. The main idea is to transform $H(k)$ by means of invertible polynomial matrices into what we will call a block-diagonal $q$ reduced form, from which the Newton polygon and the Newton polynomials can be immediately retrieved. If $H(k)$ is already in $q$-reduced form, only the first $q+1$ terms of this matrix series will be used to reach approximation order $q$. Otherwise, a lazy evaluation process allows the block-diagonal $q$-reduction process to use only a finite minimum number of terms of the matrix series. It is also shown that when $H(k)$ is symmetric then approximations of the perturbed eigenvectors are contained into the transformation matrices. The resulting algorithm has been implemented in a MAPLE package called pert.

We begin our paper presenting the physics of the problem and the $k p$-perturbation theory in Section 2.1. We then review the way the perturbed eigenelements are usually computed in Section 2.2, illustrating the method with the modified Kane matrix. Section 3 covers the more general symmetric perturbed eigenvalue problem in the case of analytic perturbations. In Section 3.1 we review how this problem can be solved algorithmically using computer algebra. Section 3.2 addresses the classical algebraic approach based on the computation of the characteristic polynomial, and recalls how this allows to draw the Newton polygon. MAPLE examples are worked out in Section 3.3. Section 4 presents our purely matricial method. Section 4.1 states the main property of the $q$-reduced form in terms of the Newton polygon and of the Newton polynomials. In Section 4.2 we provide some details about the approximation of perturbed eigenvectors, yielding our so-called " $q$-reduced form-enhanced algorithm" (Section 4.3). Finally, Section 4.4 illustrates the use of the pert package by providing an approximated diagonalization of the modified Kane matrix.

\section{A perturbed eigenvalue problem in Quantum Physics}

This section is devoted to the derivation of a symmetric perturbed eigenvalue problem from the Schrödinger equation in the context of semi-conductors. Projecting that equation on a finite-dimension vector space yields the modified Kane Matrix [5, 8]. We concentrate on the computation of the expansions of its eigenelements.

\subsection{On the kp-perturbation theory}

We aim at determining the states of a particle trapped in a quantum well, defined by the juxtaposition of semi-conductors (SC's). Physically, that means that the particle is 
surrounded by a potential $V=V_{\sim}+V_{\sqcup}$, where $V_{\sim}(x)$ is periodic, reflecting the crystalline structure of the solid. The potential $V_{\sqcup}(x)$ is a confining potential, typically

$$
V_{\sqcup}(x)= \begin{cases}0 & \text { if } x \notin\left[-x_{0}, x_{0}\right] \\ -V_{0} & \text { otherwise }\end{cases}
$$

with $x_{0}$ and $V_{0}$ positive constants.

We note $\mathcal{H}_{0}$ the Hamiltonian operator

$$
\begin{aligned}
\mathcal{H}_{0}: L^{2}(\mathbb{R}) & \longrightarrow L^{2}(\mathbb{R}) \\
u(x) & \longmapsto\left(\frac{p^{2}}{2 m_{0}}+V_{\sim}(x)\right) u(x) \quad\left(p=-i \hbar \frac{d}{d x}\right) .
\end{aligned}
$$

For the particle, the monodimensional stationary Schrödinger equation is therefore

$$
\left(\mathcal{H}_{0}+V_{\sqcup}\right) u=\lambda u,
$$

where $\lambda$ is the energy of the particle (eigenvalue of $\mathcal{H}_{0}+V_{\sqcup}$ ) and $u(x)$ is its wavefunction (associated eigenvector).

A classical method to solve the eigenvalue problem (2) may be found in [8], in the context of the

Assumption 1 The lowest eigenvalue of $\mathcal{H}_{0}+V_{\sqcup}$ is in the neighborhood of the lowest eigenvalue of $\mathcal{H}_{0}$.

Under this assumption a first step is to work on the eigenelements of $\mathcal{H}_{0}=p^{2} / 2 m_{0}+V_{\sim}$.

$V_{\sim}$ being periodic, the Bloch theorem may be applied: the eigenfunctions of $\mathcal{H}_{0}$ are of the form

$$
v_{k}(x)=e^{i k x} \psi(x)
$$

with $k$ any real number and $\psi(x)$ a function of $x$ with the same periodicity as the periodicity of the crystal. The problem being periodic, we restrict the study of $v_{k}$ to the first Brillouin zone : we want to describe the behavior of $v_{k}$ (and its associated eigenvalue $\Lambda_{k}$ ) when $k$ is in some neighborhood of zero.

According to (3), one has

$$
\frac{p^{2}}{2 m_{0}} v_{k}(x)=e^{i k x}\left(\frac{p^{2}}{2 m_{0}}+\frac{\hbar}{m_{0}} k p+\frac{\hbar^{2}}{2 m_{0}} k^{2}\right) \psi(x)
$$

and equation $\mathcal{H}_{0} v_{k}=\Lambda_{k} v_{k}$ becomes

$$
\mathcal{H}_{k} \psi=\Lambda_{k} \psi
$$

with

$$
\mathcal{H}_{k}=\mathcal{H}_{0}+k_{0}^{2}+\frac{\hbar}{m_{0}} k p \quad k_{0}^{2}=\frac{\hbar^{2}}{2 m_{0}} k^{2} .
$$

Operator $\mathcal{H}_{k}$ can thus be seen as a perturbation of operator $\mathcal{H}_{0}+k_{0}^{2}$ by $k p$. To deal with problem (4) we assume that we know an orthonormal basis $\left\{\psi_{i}\right\}_{i=1 \ldots n}$ of $n$ eigenfunctions at $k=0$ (associated with the $n$ eigenvalues at $k=0$ ) of operator $p^{2} / 2 m+V_{\sim}$ that are of fixed parity. (The orbitals may typically be s- or p-like.) This basis is chosen by the physicist depending on the description of the crystal he wants to give. 
We then project operator $\mathcal{H}_{k}$ on the finite dimension vector space generated by the $\psi_{i}$ 's

$$
\left\langle\psi_{i} \mid \mathcal{H}_{k} \psi_{j}\right\rangle=\lambda_{j} \delta_{i j}+k_{0}^{2} \delta_{i j}+\frac{\hbar}{m_{0}} k\left\langle\psi_{i} \mid p \psi_{j}\right\rangle \quad(i, j=1, \ldots, n) .
$$

Taking $V_{\sqcup}$ into account is simply adding a $Q_{i j}=\left\langle\psi_{i} \mid V_{\sqcup} \psi_{j}\right\rangle$ term to $\left\langle\psi_{i} \mid \mathcal{H}_{k} \psi_{j}\right\rangle$.

Writing $P_{i j}=\frac{\hbar}{m_{0}}\left\langle\psi_{i} \mid p \psi_{j}\right\rangle$ and using the parity of the $\psi_{i}$ 's which yields $P_{i i}=0 \forall i$ and $P_{j i}=\bar{P}_{i j} \forall i \neq j$, we get the $n \times n$ symmetric matrix

$$
H(k)=Q+\left[\begin{array}{ccc}
\lambda_{1}+k_{0}^{2} & & \left(k P_{i j}\right) \\
& \ddots & \\
\left(k \bar{P}_{i j}\right) & & \lambda_{n}+k_{0}^{2}
\end{array}\right] .
$$

whose eigenelements are to be computed: we are therefore led to the diagonalization of a matrix depending on a small parameter $k$.

\section{D. Computation of the eigenelements}

The usual way the eigenvalue expansions of $H(k)$ are computed is to use the Schrödinger perturbation formulas. The effective mass concept then only allows to approximate the perturbed eigenvectors numerically, but not to approximate their $k$-expansions.

The second order perturbation formula, obtained by Schrödinger, gives ([3], [2] p. 103)

$$
\lambda_{i}(k)=\lambda_{i}+\sum_{j \neq i} \frac{H_{i j}^{2}}{\lambda_{i}-\lambda_{j}} k^{2}+o\left(k^{2}\right) .
$$

This allows to simply derive from the matrix $H(k)$ the expressions for the eigenvalues. However, to be valid, (6) must satisfy

Assumption $2([10]) \forall i \neq j\left|H_{i j}\right|<\left|\lambda_{i}-\lambda_{j}\right|$.

One can show that this condition holds for the matrix $H(k)$ if and only if the constant matrix $Q$ is disregarded, i.e. the confining potential $V_{\sqcup}(x)$ is neglected in the Hamiltonian. For example, using this method and disregarding $Q$, Kane [8] gets for SC's GaAs and AlAs the matrix $H(k)$ (modified by Fishman [5])

$$
\left[\begin{array}{cccccccc}
\frac{3}{2}+a_{1} k^{2} & 0 & a_{2} k & 0 & a_{3} k & 0 & 0 & 0 \\
0 & \frac{3}{2}+a_{1} k^{2} & 0 & a_{2} k & 0 & -a_{3} k & 0 & 0 \\
a_{2} k & 0 & a_{4} k^{2} & 0 & a_{5} k^{2} & 0 & 0 & 0 \\
0 & a_{2} k & 0 & a_{4} k^{2} & 0 & -a_{5} k^{2} & 0 & 0 \\
a_{3} k & 0 & a_{5} k^{2} & 0 & -\frac{17}{50}+a_{6} k^{2} & 0 & 0 & 0 \\
0 & -a_{3} k & 0 & -a_{5} k^{2} & 0 & -\frac{17}{50}+a_{6} k^{2} & 0 & 0 \\
0 & 0 & 0 & 0 & 0 & 0 & a_{7} k^{2} & 0 \\
0 & 0 & 0 & 0 & 0 & 0 & 0 & a_{7} k^{2}
\end{array}\right]
$$

with

$$
a_{1}=\frac{11049}{1000}=11.05 \quad a_{2}=\frac{87368}{10751}=8.13 \quad a_{3}=\frac{119299}{20761}=5.75
$$


$a_{4}=\frac{6225377}{2941123}=2.12 \quad a_{5}=\frac{48704}{5729}=8.50 \quad a_{6}=-\frac{817954}{210019}=3.89 \quad a_{7}=-\frac{4953}{500}=9.91$

In [5] Fishman computes the six nontrivial eigenvalue expansions of the modified Kane matrix (7) up to order 2 using the Schrödinger formula (6)

$$
\begin{aligned}
& \lambda_{1,2}(k)=1.5+73.02 k^{2}+o\left(k^{2}\right), \\
& \lambda_{3,4}(k)=-41.91 k^{2}+o\left(k^{2}\right), \\
& \lambda_{5,6}(k)=-0.34-21.84 k^{2}+o\left(k^{2}\right) .
\end{aligned}
$$

One could also compute the perturbed eigenvectors up to order 2 with some appropriate Schrödinger perturbation formulas. However, while highly influencing them, the confining potential $V_{\sqcup}$ has been so far disregarded, because it prevents $H(k)$ from fulfilling Assumption 2.

The former computation of the eigenvalues will therefore be used only as a starting point. The effective mass method is used to go beyond: neglecting the confining potential means that we are actually treating the case of a free particle in a periodic potential. Besides, the spectrum of the Hamiltonian corresponding to a free particle is

$$
\lambda(k)=-\frac{\hbar^{2}}{2 m_{0}} k^{2} .
$$

Thus, comparing (9) with, say, (8a), one may infer that the particle in the periodic potential behaves as if it were a free particle of mass $m_{0}^{*}$ defined by

$$
73.02 k^{2}=\frac{-\hbar^{2}}{2 m_{0}^{*}} k^{2} .
$$

$m_{0}^{*}$ is called the effective mass of the particle in the SC's (for a given energy). Now, to compute an energy and its corresponding wavefunction of that particle in the confining potential one has to solve another Schrödinger equation

$$
\left(\frac{p^{2}}{2 m_{0}^{*}}+V_{\sqcup}(x)\right) v(x)=\lambda^{*} v(x) .
$$

This second equation may be solved by any classical numerical method, among which variational techniques, or by discretizing the equation and using Lanczos-like algorithms to compute the eigenelements of the resulting matrix. Thus, the effective mass concept allows to isolate the action of the periodic potential (taken into account by $m_{0}^{*}$ defined via the $k p$-theory) from the action of the confining potential. However, it will require the numerical resolution of as many Schrödinger equations of type (10) as the number of distinct eigenvalues of $H(k)$.

Remark: The only reason why we do not take $Q$ into account directly into the perturbation formulas is Assumption 2. Therefore, another perturbative approach than Schrödinger's method, which would not rely on some numerical approximations, could allow to directly take the matrix $Q$ (that is to say, the confining potential) into account, without having to resort to the effective mass theory. 
In the sequel we will therefore focus on the search for eigenvalue and eigenvector expansions of the matrix $H(k)$ (with or without the $Q$ matrix) through such direct methods.

\section{The symmetric perturbed eigenvalue problem}

We first recall some notions about the symmetric perturbed eigenvalue problem in the case of analytic perturbations (the small parameter $k$ will now be denoted by $\varepsilon$ ). We then briefly explain why the main problem actually consists in building a Newton polygon, which traditionnally results from the computation of the characteristic polynomial of the matrix. Some existing MaPLE functionalities, based on such a computation, are also presented.

\subsection{An algorithmic view of the problem}

Let $F$ denote the ring of complex analytic functions of the real variable $\varepsilon$ in some neighborhood of zero. Let $A(\varepsilon) \in F^{n \times n}$. The matrix $A(\varepsilon)$ is said to be analytic (in $\varepsilon=0)$ and can be written

$$
A(\varepsilon)=\sum_{k=0}^{\infty} A_{k} \varepsilon^{k} \quad\left(\forall k \geq 0, A_{k} \in \mathbb{C}^{n \times n}\right) .
$$

We want to compute the eigenelements of $A(\varepsilon)$, i.e. $\lambda(\varepsilon)$ and $\psi(\varepsilon) \neq 0$ satisfying

$$
A(\varepsilon) \psi(\varepsilon)=\lambda(\varepsilon) \psi(\varepsilon)
$$

where $A_{0}$ is supposed not to vanish identically. Moreover, $\varepsilon$ may be seen as a small perturbative parameter and the analytic perturbation theory for linear operators [2] allows to consider $A(\varepsilon)$ as an analytic perturbation of $A_{0}$. It is then well known ([2] p.351, [9] p.65) that each eigenvalue or eigenvector of $A(\varepsilon)$ admits an expansion in fractional powers of $\varepsilon$ (Puiseux series), whose zero-th order term is an eigenvalue or an eigenvector of the unperturbed matrix $A_{0}$.

For our application, $A(\varepsilon)$ is supposed to be symmetric for all $\varepsilon$ real, that is $A^{*}(\varepsilon)=A(\varepsilon), \forall \varepsilon \in \mathbb{R}$. In this case, the eigenelements of $A(\varepsilon)$ are analytic functions of $\varepsilon([2]$ p.21, [9] p.120). Thus, all the powers of $\varepsilon$ involved in these expansions are integers:

$$
\lambda(\varepsilon)=\lambda_{0}+\sum_{k=1}^{\infty} \lambda_{k} \varepsilon^{k} \quad \psi(\varepsilon)=\psi_{0}+\sum_{k=1}^{\infty} \psi_{k} \varepsilon^{k}
$$

with $\lambda_{0}$ and $\psi_{0}$ such that $A_{0} \psi_{0}=\lambda_{0} \psi_{0}$. We call valuation of the eigenvalue $\lambda(\varepsilon)$ the smallest power of $\varepsilon$ that appears in the expansion of $\lambda(\varepsilon)$. This definition also applies to the eigenvector $\psi(\varepsilon)$. To compute an approximation of $\lambda(\varepsilon)$ and $\psi(\varepsilon)$ up to order $q \in \mathbb{N}$, one rewrites $\lambda(\varepsilon)$ as

$$
\lambda(\varepsilon)=\mu \varepsilon^{\beta}+o\left(\varepsilon^{\beta}\right), \quad \mu \in \mathbb{C}, \beta \in \mathbb{N} .
$$

This yields the following sketch of an algorithm: 
1.1 Compute $\mu$ and $\beta(\beta \leq q)$;

1.2 While $\beta<q$ call step 1.1 with $A(\varepsilon)-\mu \varepsilon^{\beta} I$;

The result is $\lambda^{(q)}(\varepsilon)$ such that $\lambda(\varepsilon)=\lambda^{(q)}(\varepsilon)+o\left(\varepsilon^{q}\right)$. A corresponding eigenvector (of valuation zero) may then be approximated up to order $q$ by solving

$$
\left(A(\varepsilon)-\lambda^{(q)}(\varepsilon) I\right) \psi^{(q)}(\varepsilon)=o\left(\varepsilon^{q}\right)
$$

or, equivalently, by taking the step

1.3 Solve $\sum_{j=0}^{k}\left(A_{j}-\lambda_{j}^{(q)} I\right) \psi_{k-j}^{(q)}=0 \quad(\forall k=0, \ldots, q)$.

The main difficulty is thus to determine the leading exponent $\beta$ and the associated leading coefficient $\mu$ for each eigenvalue of $A(\varepsilon)$.

\subsection{The classical algebraic approach}

Let $\chi(\varepsilon, \lambda)=\operatorname{det}(\lambda I-A(\varepsilon))$ be the characteristic polynomial of $A(\varepsilon)$. It satisfies

$$
\chi(\varepsilon, \lambda)=\lambda^{n}+\alpha_{1}(\varepsilon) \lambda^{n-1}+\cdots+\alpha_{n-1}(\varepsilon) \lambda+\alpha_{n}(\varepsilon)
$$

and consists of a polynomial in $\lambda$, with analytic coefficients

$$
\alpha_{i}(\varepsilon)=\hat{\alpha}_{i} \varepsilon^{a_{i}}+o\left(\varepsilon^{a_{i}}\right) \quad(i=1, \ldots, n) .
$$

where $a_{i}$ is the leading exponent and $\hat{\alpha}_{i}$ the leading coefficient of $\alpha_{i}(\varepsilon)$ (i.e. $\hat{\alpha}_{i} \neq 0$ and no term of order lower than $a_{i}$ appears in the expansion of $\alpha_{i}(\varepsilon)$ ). The solutions of $\chi(\varepsilon, \lambda)=0$ are the eigenvalues $\lambda(\varepsilon)=\mu \varepsilon^{\beta}+o\left(\varepsilon^{\beta}\right)$ of $A(\varepsilon)$. An easy way to find the first-order term $\mu \varepsilon^{\beta}$ is to associate with $\chi(\varepsilon, \lambda)$ its so-called Newton polygon (also known as Puiseux-Newton diagram and noted $\mathcal{N}$ ). It is defined as follows. We plot the values $a_{i}$ versus $i$ for $i=1, \ldots, n$ together with the point $(0,0)$ corresponding to $\lambda^{n}$ (if $\alpha_{i}(\varepsilon)=0$, the corresponding point is disregarded). Then we draw the segments on the lower boundary of the convex hull of the plotted points. These segments constitute the Newton polygon $\mathcal{N}$ associated with $\chi(\varepsilon, \lambda)$ or, equivalently, the Newton polygon of $A(\varepsilon)$ (see [12] for examples in the general nonsymmetric case). Let $\mathcal{S}_{s}$ be the segment of $\mathcal{N}$ whose slope is $s\left(s \in \mathbb{Q}_{+}\right)$. One can associate with $\mathcal{S}_{s}$ the so-called Newton polynomial $p_{s}(\lambda)$ such that $[2,12]$

$$
p_{s}(\lambda)=\sum_{\left(i, a_{i}\right) \in \mathcal{S}_{s}} \hat{\alpha}_{i} \lambda^{n-i} .
$$

The knowledge of the Newton polynomials is crucial, because they have the following property.

Proposition 1 ([2] §A7, [12] §3) $A(\varepsilon)$ has exactly $m$ eigenvalues of the form $\lambda(\varepsilon)=$ $\mu \varepsilon^{\beta}+o\left(\varepsilon^{\beta}\right)$ iff $\mu$ is a root of the Newton polynomial $p_{\beta}(\lambda)$ of multiplicity $m$.

In particular, that means that the slopes of the Newton polygon of $A(\varepsilon)$ are exactly the exponents of the leading terms of the perturbed eigenvalues. Besides, $A(\varepsilon)$ being symmetric, then, according to (11), its Newton polygon consists of integer slopes only. 


\section{Examples}

(i) Let $A(\varepsilon)$ be the following singular matrix of dimension 5:

$$
A(\varepsilon)=\left[\begin{array}{cccc}
1+\varepsilon & \varepsilon & \varepsilon & \\
\varepsilon & \varepsilon^{2} & & \\
\varepsilon & & \varepsilon^{2}+\varepsilon^{3} & \\
& & & \varepsilon^{3}
\end{array}\right] .
$$

One has $\chi(\varepsilon, \lambda)=\lambda^{5}-\left(1+\varepsilon+2 \varepsilon^{2}+2 \varepsilon^{3}\right) \lambda^{4}+\left(4 \varepsilon^{3}+3 \varepsilon^{4}+3 \varepsilon^{5}+\varepsilon^{6}\right) \lambda^{3}+\left(\varepsilon^{4}-\varepsilon^{5}-\right.$ $\left.4 \varepsilon^{6}-2 \varepsilon^{7}-\varepsilon^{8}\right) \lambda^{2}-\left(\varepsilon^{7}-\varepsilon^{8}-\varepsilon^{9}\right) \lambda$. The Newton polygon $\mathcal{N}$ of $A(\varepsilon)$ is represented in figure 1 and (13) gives three Newton polynomials

$$
p_{0}(\lambda)=\lambda^{4}(\lambda-1) \quad p_{2}(\lambda)=-\lambda^{2}\left(\lambda^{2}-1\right) \quad p_{3}(\lambda)=\lambda(\lambda-1) .
$$

Proposition 1 says then that the eigenvalues of $A(\varepsilon)$ belong to

$$
\left\{1+o(1), \quad \pm \varepsilon^{2}+o\left(\varepsilon^{2}\right), \quad \varepsilon^{3}+o\left(\varepsilon^{3}\right)\right\} .
$$

The infinite slope of $\mathcal{N}$ corresponds to the (simple) eigenvalue 0 of $A(\varepsilon)$.

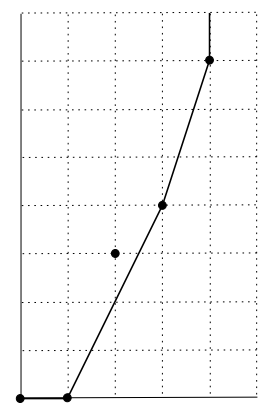

Figure 1. Newton polygon for example (i).

(ii) Let $A(\varepsilon)$ be the modified Kane matrix (7). The computation of $\chi(\varepsilon, \lambda)$ gives

$$
\begin{aligned}
\chi(\varepsilon, \lambda)=\lambda^{8} & +\left(\hat{\alpha}_{1}+o(1)\right) \lambda^{7}+\left(\hat{\alpha}_{2}+o(1)\right) \lambda^{6}+\left(\hat{\alpha}_{3}+o(1)\right) \lambda^{5}+\left(\hat{\alpha}_{4}+o(1)\right) \lambda^{4} \\
& +\left(\hat{\alpha}_{5} \varepsilon^{2}+o\left(\varepsilon^{2}\right)\right) \lambda^{3}+\left(\hat{\alpha}_{6} \varepsilon^{4}+o\left(\varepsilon^{4}\right)\right) \lambda^{2}+\left(\hat{\alpha}_{7} \varepsilon^{6}+o\left(\varepsilon^{6}\right)\right) \lambda+\hat{\alpha}_{8} \varepsilon^{8}+o\left(\varepsilon^{8}\right)
\end{aligned}
$$

with $\hat{\alpha}_{i}, i=1, \ldots, 8$, some nonzero rational numbers. Hence the Newton polygon in figure 2 and two Newton polynomials $p_{0}(\lambda)=\lambda^{4}\left(\lambda^{4}+\hat{\alpha}_{1} \lambda^{3}+\hat{\alpha}_{2} \lambda^{2}+\hat{\alpha}_{3} \lambda+\hat{\alpha}_{4}\right)$ and $p_{2}(\lambda)=\hat{\alpha}_{4} \lambda^{4}+\hat{\alpha}_{5} \lambda^{3}+\hat{\alpha}_{6} \lambda^{2}+\hat{\alpha}_{7} \lambda+\hat{\alpha}_{8}$ whose nonzero roots are to be computed (for the moment, all we can say is that $A(\varepsilon)$ has four eigenvalues of valuation 0 and four eigenvalues of valuation 2).

In practice, computing $\chi(\varepsilon, \lambda)$ allows to build the Newton polygon of $A(\varepsilon)$, to compute its Newton polynomials according to (13) and hence to get $\mu$ and $\beta$ for every eigenvalue of $A(\varepsilon)$. Reinserting $\lambda+\mu \varepsilon^{\beta}$ into the expression of $\chi(\varepsilon, \lambda)$ will lead to the next terms of the Puiseux series. More precisely, steps 1.1 and 1.2 of the algorithm 


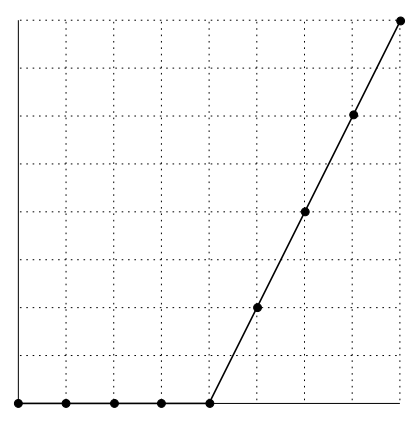

Figure 2. Newton polygon for example (ii): the modified Kane matrix.

become

2.0 Compute $\chi(\varepsilon, \lambda)=\operatorname{det}(\lambda I-A(\varepsilon))$ and $v \leftarrow 0$;

2.1 - Draw the segments of $\mathcal{N}$ of slope $s \in[v, q]$ and compute the associated $p_{s}$ 's,

- The nonzero roots $\mu_{s j}$ of the $p_{s}$ 's give all the first-order terms $\mu_{s j} \varepsilon^{s}$;

2.2 For each $\mu_{s j} \varepsilon^{s}$ do

if $s<q$ then $v \leftarrow v+1$ and call step 2.1 with $\chi\left(\varepsilon, \lambda+\mu_{s j} \varepsilon^{s}\right)$;

Steps 2.1 and 2.2 are traditionally referred to as the Newton method to solve algebraic equations. The first effective use of this method in computer algebra is due to Duval and may be found in [4]. The next section illustrates how this approach can be used in practice, and briefly discusses its limitations.

\subsection{Using some MAPLE functionalities}

The MAPLE built-in function linalg[eigenvals] computes $\chi(\varepsilon, \lambda)=\operatorname{det}(\lambda I-A(\varepsilon))$ and tries to find the exact solutions $\lambda(\varepsilon)$ of the algebraic equation $\chi(\varepsilon, \lambda)=0$. Unless $\chi(\lambda, \varepsilon)$ factors (with respect to $\lambda$ ) into linear or quadratic factors, either the exact roots are messy (cubic or quartic factors), or there is no way to express them in terms of exact radicals (quintic or higher degree factors). In both cases, the results produced will be of very limited use, even for matrices of small dimension $(<5)$ and perturbations of small degree $(=1)$ (e.g. see [11]). Using linalg [eigenvects] or the existing functions computing the symbolic Jordan canonical form to get both the perturbed eigenvalues and eigenvectors of $A(\varepsilon)$ would be worse. For problems of higher dimension, such as the modified Kane matrix, and more generally in the case of polynomial perturbations, one may use the puiseux function [15]. The puiseux function is an efficient implementation of steps 2.1 and 2.2. It computes the Puiseux expansions (for $\varepsilon$ in some given neighborhood) of the roots of the irreducible factors of $\chi(\varepsilon, \lambda)$ up to any prescribed order $q$. That means that it allows to approximate the eigenvalues of $A(\varepsilon)$ up to order $q$. Here is an example with the modified Kane matrix (7), $\varepsilon$ in some neighborhood of zero and $q=2$. The berkosam procedure implements an improved version of the Berkowitz algorithm 
to compute the characteristic polynomial of $A(\varepsilon)$. It is faster than linalg [charpoly] ([1] p.29).

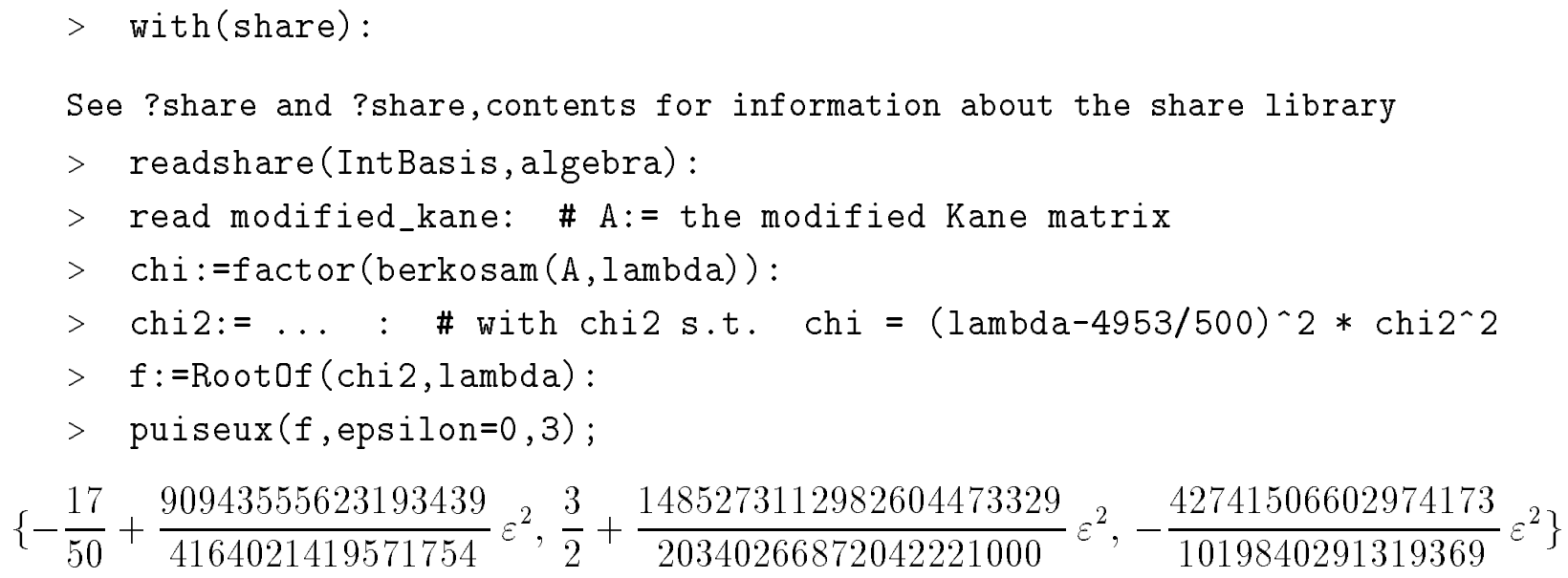

Therefore, expansions (7) can be computed automatically. Moreover, increasing $q$ will lead to more accurate approximations of the perturbed eigenvalues.

However, in the case of analytic perturbations, the puiseux command will fail to give such approximations. Moreover, this algebraic approach demands the computation (and the factorization) of the characteristic polynomial of $A(\varepsilon)$, which is not optimal. Indeed, even in the case of polynomial perturbations, the Newton polynomials required to approximate the eigenvalues of $A(\varepsilon)$ up to order $q$ depend only on a few terms of $A(\varepsilon)$. Hence, the characteristic polynomial $\chi(\lambda, \varepsilon)$ of $A(\varepsilon)$ contains in general much more information than these required to determine all the Newton polynomials needed for our approximation. Since the computation of $\chi(\lambda, \varepsilon)$ may become dramatically time consuming when the size of the matrix and/or the degree of the perturbation increase(s), we want to avoid such a computation, i.e. avoid step 2.0, and use as few terms of $A(\varepsilon)$ as possible to perform steps 2.1 and 2.2 .

Another drawback of the algebraic approach is that it says nothing about the perturbed eigenvectors and some nontrivial additionnal work (taking step 1.3, see equation (5)) will be necessary to actually diagonalize $A(\varepsilon)$. Thus, the computer algebra functionalities used so far solve the symmetric perturbed eigenvalue problem neither efficiently enough nor completely.

\section{Empowering the classical approach with the $q$-reduced form}

The $q$-reduced form of an analytic matrix is presented, which allows to recover both the Newton polygon and the Newton polynomials, without computing the whole characteristic polynomial of the matrix. Moreover, in the symmetric case, it grants approximations for the perturbed eigenvectors. The resulting algorithm has been used to diagonalize the modified Kane matrix. 


\subsection{The q-reduced form}

We use $\lambda(\varepsilon)$ to denote the eigenvalues of $A(\varepsilon)$. If we restrict ourselves to $A_{0}$, all we can get is the constant term of the expansions of the $\lambda(\varepsilon)$ 's. That classical result (see e.g. $[9$, p.65]) is exactly expressed by Proposition 1 for $\beta=0$. Hence, if 0 is a slope of $\mathcal{N}, A_{0}$ allows to compute the first Newton polynomial and $p_{0}(\lambda)=\operatorname{det}\left(\lambda I-A_{0}\right)$. But for the eigenvalues whose nonzero first-order term has a leading exponent greater than zero, all we can say is that $\lambda(\varepsilon)=o(1)$. To get this first-order term, say $\mu \varepsilon^{s}\left(s \in \mathbb{N}^{*}\right.$ is a slope of $\left.\mathcal{N}\right)$, one has to know $p_{s}(\lambda)$ for which one should use more terms than $A_{0}$. This can be done as described below.

Let us denote by $v_{i}$ the valuation of the $i$-th column of $A(\varepsilon)$, i.e. the smallest power of $\varepsilon$ appearing in the entries of this column $\left(v_{i}=+\infty\right.$ in the case of a zero column). Let

$$
D_{s}(\varepsilon)=\operatorname{diag}\left(\varepsilon^{-\min \left(0, v_{i}-s\right)}\right)_{1 \leq i \leq n} .
$$

$D_{s}(\varepsilon)=D_{s, 0}+D_{s, 1} \varepsilon+\cdots$ is an $n \times n$ invertible polynomial matrix of valuation lower or equal to $s$. Consequently, there exists $N_{s}(\varepsilon)=N_{s, 0}+N_{s, 1} \varepsilon+\cdots \in F^{n \times n}$ such that $A(\varepsilon)$ can be factored

$$
A(\varepsilon)=\varepsilon^{s} N_{s}(\varepsilon) D_{s}^{-1}(\varepsilon) \text {. }
$$

Defining

$$
\theta_{s}(\lambda)=\operatorname{det}\left(N_{s, 0}-\lambda D_{s, 0}\right)
$$

one has

Proposition $2\left([7]\right.$ p.123) Suppose that $\theta_{s} \not \equiv 0$. Then $A(\varepsilon)$ has exactly $m$ eigenvalues $\lambda(\varepsilon)=\mu \varepsilon^{s}+o\left(\varepsilon^{s}\right)$ iff $\mu$ is a root of $\theta_{s}(\lambda)$ of multiplicity $m$.

That means that if $\theta_{s} \not \equiv 0, \theta_{s}$ equals the Newton polynomial $p_{s}$ within a monomial in $\lambda$. The integer $s$ is the slope of a segment of $\mathcal{N}$ iff $\theta_{s}$ has at least one nonzero root. Moreover, $\theta_{s}$ depends only on $A_{0}, \ldots, A_{s}$ by construction. With $N_{0}(\varepsilon)=A(\varepsilon)$ and $D_{0}(\varepsilon)=I$, one finds the classical case $s=0$ again and $\theta_{0}(\lambda)=\operatorname{det}\left(A_{0}-\lambda I\right)=(-1)^{n} p_{0}(\lambda)$. Hence, if

$$
\theta_{s} \not \equiv 0 \quad \text { for all slope } s \text { of } \mathcal{N}
$$

all the Newton polynomials of $A(\varepsilon)$ are given by (15). In the worst case, the computation will require the first $\kappa+1$ terms of $A(\varepsilon)$ where $\kappa \in \mathbb{N}$ stands for the largest slope of $\mathcal{N}$. Otherwise, we propose to transform $A(\varepsilon)$ into a particular form so that (16) is satisfied.

In [7] we define the so-called $q$-reduced form of a matrix $A(\varepsilon)$ depending on parameter $\varepsilon$. It is an adaptation to the algebraic case of the Moser- and super-reduced forms defined for linear differential systems $[13,6]$ and used for their symbolic resolution [14]. The equivalence used is similarity in the field of meromorphic matrices: $A(\varepsilon)$ and $B(\varepsilon)$ are similar if and only if there exists a meromorphic matrix $T(\varepsilon)$ invertible $\forall \varepsilon \neq 0$ so that $T^{-1}(\varepsilon) A(\varepsilon) T(\varepsilon)=B(\varepsilon)$.

By definition, $A(\varepsilon)$ is in $q$-reduced form if it has a minimum number of columns of each valuation lower or equal to $q-1$ among all the matrices similar to $A(\varepsilon)$. An effective algorithm to compute a polynomial similarity transformation $P(\varepsilon)$ such 
that $P^{-1}(\varepsilon) A(\varepsilon) P(\varepsilon)$ is in $q$-reduced form is also given. The practical interest of computing such a form comes from the following property (reformulated here in the case of symmetric analytic matrices).

Proposition 3 ([7] p.123) Let $q \in I N$ and $A(\varepsilon) \in F^{n \times n}$ be symmetric $\forall \varepsilon \in \mathbb{R}$. $A(\varepsilon)$ is in $q$-reduced form iff $\theta_{s} \not \equiv 0, \forall s \leq q$.

Computing a $q$-reduced form of $A(\varepsilon)$ with $q \geq \kappa$ therefore allows to get all the Newton polynomials. In practice, the value of $\kappa$ being unknown, $q$ will stand for the approximation order and may be lower than $\kappa$.

\subsection{Approximation of eigenvectors}

Lemma 1 ([7] p.125) Let $q \in I N$ and $A(\varepsilon) \in F^{n \times n}$ be symmetric $\forall \varepsilon \in I R$. There exists a similarity transformation $T(\varepsilon)$ such that $T^{-1}(\varepsilon) A(\varepsilon) T(\varepsilon)$ is in q-reduced form and block-diagonal. Besides, the Newton polygon of each block consists of (part of) a segment of integer slope.

Notice that although $T^{-1}(\varepsilon)$ may have poles, $T^{-1}(\varepsilon) A(\varepsilon) T(\varepsilon)$ will not, because of the definition of a $q$-reduced form. Indeed, to compute such a form, we only allow to decrease the number of columns of $A(\varepsilon)$ with valuation lower than $q>0$, which contradicts any pole introduction.

Lemma 1 means that the Newton polygon "splits" completely and that the firstorder terms of the eigenvalues of $A(\varepsilon)$ can be separated:

$$
T^{-1}(\varepsilon) A(\varepsilon) T(\varepsilon)=\operatorname{diag}\left(\cdots, A_{s}^{1}(\varepsilon), \cdots, A_{s}^{r_{s}}(\varepsilon), \cdots\right)
$$

where $A_{s}^{j}(\varepsilon)$ is a block whose all eigenvalues are $\mu_{s, j} \varepsilon^{s}+o\left(\varepsilon^{s}\right)\left(j=1, \ldots, r_{s}\right.$ and $\left.\mu_{s i} \neq \mu_{s j}, \forall i \neq j\right)$. Applying Lemma 1 up to a given order - namely, $s$ - yields the following corollary. In the symmetric case, it allows for a direct approximation of the perturbed eigenvectors of $A(\varepsilon)$.

Corollary 1 Let $j \in\left[1 . . r_{s}\right]$ be given and $B^{(s)}(\varepsilon)$ be the approximation up to order $s$ of the corresponding block $A_{s}^{j}(\varepsilon)$ given by Lemma 1. Let $m$ be the size of $A_{s}^{j}(\varepsilon)$ and $\mu=\mu_{s j}$. Then $A(\varepsilon)$ has exactly $m$ eigenvalues whose first-order term is $\mu \varepsilon^{s}$ and

$$
T^{-1}(\varepsilon)\left[A(\varepsilon)-\mu \varepsilon^{s} I\right] T(\varepsilon)=\left[\begin{array}{ccc|c}
* & \cdots & * & \\
\vdots & & \vdots & (0) \\
* & \cdots & * & \\
\hline & (0) & & B^{(s)}(\varepsilon)
\end{array}\right]+o\left(\varepsilon^{s}\right)
$$

where o( $\left.\varepsilon^{s}\right)$ denotes an $n \times n$ matrix with entries of the form o $\left(\varepsilon^{s}\right)$. Besides, if $A(\varepsilon)$ is symmetric $\forall \varepsilon \in \mathbb{R}$, then $B^{(s)}(\varepsilon)=0$.

Proof- The lefthand side of (17) is diagonalizable $\forall \varepsilon \in \mathbb{R}$. Thus the quantity $B^{(s)}(\varepsilon)+o\left(\varepsilon^{s}\right)$ in the righthand side is also diagonalizable $\forall \varepsilon \in \mathbb{R}$. 
Moreover, if $\kappa^{-}$is the smallest slope of $\mathcal{N}$, the valuation $v$ of a $q$-reduced form with $q \geq \kappa^{-}$is greater or equal to $\kappa^{-}$. Indeed, assume that $v<\kappa^{-}$. All eigenvalues have a valuation greater or equal to $\kappa^{-}$, i.e. their leading coefficients $\mu$ satisfy

$$
\begin{array}{ll} 
& \operatorname{det}\left(A_{v} \varepsilon^{v}+o\left(\varepsilon^{v}\right)-\left(\mu \varepsilon^{\kappa^{-}}+o\left(\varepsilon^{\kappa^{-}}\right)\right) I\right)=0 \\
\stackrel{\varepsilon \neq 0}{\Longrightarrow} & \operatorname{det}\left(N_{v}(\varepsilon) D_{v}^{-1}(\varepsilon)-\varepsilon^{\kappa^{-}-v}(\mu+o(1)) I\right)=0 \\
\stackrel{\varepsilon \rightarrow 0}{\Longrightarrow} & \theta_{v}(\mu)=\operatorname{det}\left(N_{v, 0}\right)=0 \quad \text { because } \kappa^{-}-v>0 .
\end{array}
$$

That means that $\theta_{v} \equiv 0, v<\kappa^{-} \leq q$, which contradicts the fact that the matrix is $q$-reduced (see Proposition 3 ).

Thus, $T^{-1}(\varepsilon) A(\varepsilon) T(\varepsilon)$ being in $q$-reduced form and because of the splitting of $\mathcal{N}$, the valuation of $B^{(s)}(\varepsilon)$ is $s$. More precisely, $B^{(s)}(\varepsilon)=\varepsilon^{s} B$ with $B$ nilpotent. Therefore,

$$
\left.\frac{B^{(s)}(\varepsilon)+o\left(\varepsilon^{s}\right)}{\varepsilon^{s}}\right|_{\varepsilon=0}=B
$$

is diagonalizable and nilpotent, i.e. $B=0$.

That implies that the last $m$ columns of $T(\varepsilon)\left(\boldsymbol{X}_{i}(\varepsilon), i=1, \ldots, m\right)$, satisfy

$$
A(\varepsilon) \boldsymbol{X}_{i}(\varepsilon)=\mu \varepsilon^{s} \boldsymbol{X}_{i}(\varepsilon)+o\left(\varepsilon^{s}\right) \quad(i=1, \ldots, m) .
$$

Hence, $\mu \varepsilon^{s}$ being the approximation up to order $s$ of $m$ eigenvalues of $A(\varepsilon)$, the $\boldsymbol{X}_{i}(\varepsilon)$ 's are approximations up to order $s-v_{i}$ of the associated exact eigenvectors $\left(v_{i} \in \mathbb{I N}\right.$ denotes the valuation of vector $\left.\boldsymbol{X}_{i}(\varepsilon)\right)$. Lastly, if $v_{i}=0, \boldsymbol{X}_{i}(\varepsilon)$ is normalized so that $A_{0} \boldsymbol{X}_{i}(0)=\lambda_{0} \boldsymbol{X}_{i}(0)$ (otherwise, use $\boldsymbol{X}_{i}(\varepsilon) / \varepsilon^{v_{i}}$ ).

\subsection{A q-reduced form-enhanced algorithm}

From an algorithmic point of view, a lazy evaluation process allows to compute a finite value $l \in \mathbb{N}$ and $L(\varepsilon), R(\varepsilon)$ polynomial matrices such that Lemma 1 holds up to approximation order $q$, i.e.

$$
L(\varepsilon)\left[A_{0}+A_{1} \varepsilon+\cdots+A_{l} \varepsilon^{l}\right] R(\varepsilon)=\operatorname{diag}\left(\cdots, A_{s}^{1,(q)}(\varepsilon), \cdots, A_{s}^{r_{s},(q)}(\varepsilon), \cdots\right)+o\left(\varepsilon^{q}\right)
$$

is in $q$-reduced form (see [14, p.160] for details about that lazy evaluation process). The technique described above leads to the following algorithm (in pseudocode)

$v \leftarrow 0 ; \quad T_{q}(\varepsilon) \leftarrow I_{n} ;$

3.1 - Apply Lemma 1 up to order $q$ to get $l, L(\varepsilon), R(\varepsilon)$ and $\operatorname{diag}\left(\cdots, A_{s}^{1,(q)}(\varepsilon), \cdots, A_{s}^{r_{s},(q)}(\varepsilon), \cdots\right)+o\left(\varepsilon^{q}\right)$ in $q$-reduced form;

- $\forall s \in[v, q]$, use (15) to deduce $\theta_{s}$ from $\operatorname{diag}\left(A_{s}^{1,(q)}(\varepsilon), \cdots, A_{s}^{r_{s},(q)}(\varepsilon)\right)$;

- The roots $\mu_{s j}$ of the $\theta_{s}$ 's give all the first-order terms $\mu_{s j} \varepsilon^{s}$;

- Update $T_{q}(\varepsilon)$ with $R(\varepsilon)$;

3.2 For each $\mu_{s j} \varepsilon^{s}$ do if $s<q$ then $v \leftarrow s+1$ and call step 3.1 with $A_{s}^{j,(q)}(\varepsilon)-\mu_{s j} \varepsilon^{s}$; 
At this point, all the eigenvalues $\lambda(\varepsilon)$ of $A(\varepsilon)$ have been approximated by some $\lambda^{(q)}(\varepsilon)$ 's up to order $q$. A finite number of terms of the series $A(\varepsilon)$ has been used and we did not need a brute force computation of its characteristic polynomial. Moreover, the transformation matrix $T_{q}(\varepsilon)$ computed so far contains the result of step 1.3 (see equation (12)). Indeed, the columns of $T_{q}(\varepsilon)$ are approximations up to order (at most) $q$ of the eigenvectors associated with the $\lambda(\varepsilon)$ 's. Therefore, approximations of both the perturbed eigenvalues and eigenvectors can be computed simultaneously using successive appropriate $q$-reduced forms. The way $T_{q}(\varepsilon)$ is updated at each recursive call is detailed in [7].

\subsection{Application to the physical problem}

The algorithm presented in the previous section has been implemented in a MAPLE V R4 package called pert. The pert package contains functions pert_reduce and eigenvects. The first one performs steps 3.1 and 3.2 and the second just collects the eigenvector approximations from $T_{q}(\varepsilon)$. Moreover, this package heavily relies on the ISOLDE package. ISOLDE has been designed by E. Pflügel [14] for the symbolic resolution of linear differential systems. Both these MAPLE packages are available at

\section{http:// www-lmc.imag.fr/lmc-cf/Claude-Pierre.Jeannerod/}

What follows is a MAPLE session example to ilustrate how the algorithm proposed in Section 4.3 can be used to diagonalize the modified Kane matrix up to a given order (here $q=2$ ). Remark that pert_reduce returns for each eigenvalue approximation, both its multiplicity $m$ and the corresponding block $A_{j}^{s,(q)}(\varepsilon)$ (written Asjq in the MAPLE format). For more legibility, the option float of eigenvects may be used, which simply applies evalf (with the 10 digits MAPLE standard) to the approximate eigenvector.

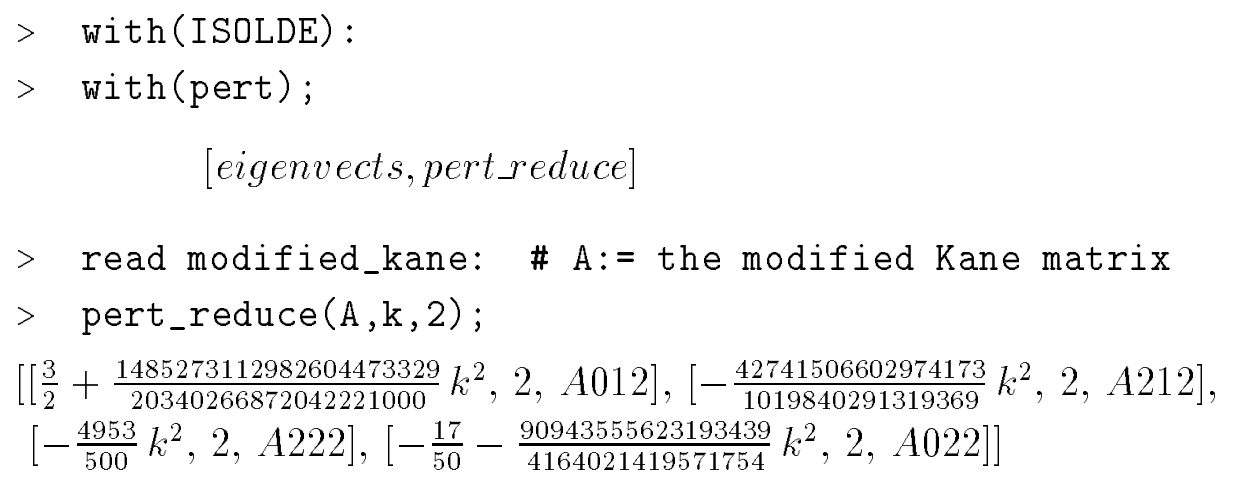

The corresponding eigenvector approximations are:

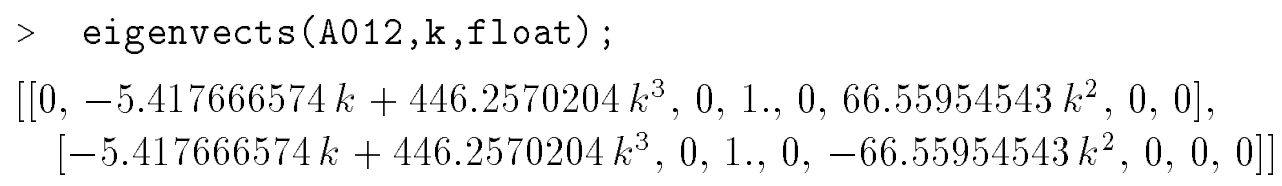




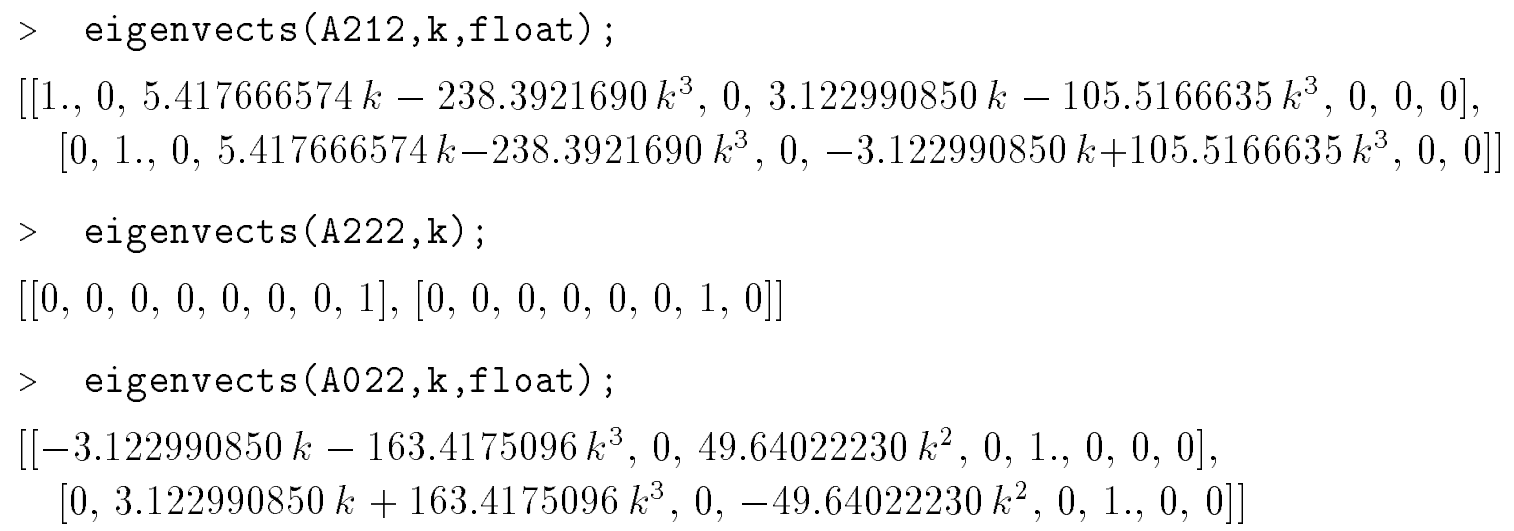

\section{Conclusion}

We have proposed a MAPLE package to solve the symmetric perturbed eigenvalue problem in the case of analytic matrices. When compared to the classical algebraic method, our approach turns out to be more efficient in the sense that it does not require the computation of the characteristic polynomial of the matrix series. This is important when dealing with large matrices $(n=50)$ and/or high degree or analytic perturbations and when the approximation order is small, namely $q=2$. Besides, it solves the problem completely by simultaneously approximating both the perturbed eigenvalues and eigenvectors up to any order. The pert package will be used to diagonalize various modified Kane matrices which differ by matrix $Q$ (see equation (5)).

\section{Acknowledgments}

We thank Guy Fishman and Ladislav Kocbach for their interest and useful suggestions. Gilles Villard brought [1] to our attention, and Eckhard Pflügel allowed us to extend the use of ISOLDE to algebraic problems.

\section{References}

[1] Abdeljaoued J 1997 The Berkowitz algorithm, MAPLE and computing the characteristic polynomial in an arbitrary commutative ring MapleTech 4 (3) 21-32

[2] Baumgärtel H 1985 Analytic perturbation theory for matrices and operators (Basel: Birkhauser Verlag)

[3] Cohen-Tannoudji C 1973 Mécanique quantique, volumes I et II (Hermann)

[4] Duval D 1987 Diverses questions relatives au calcul formel avec nombres algébriques Thèse d'Etat, Faculté des Sciences de Grenoble

[5] Fishman G 1997 Quasi-cube et Würtzite: application au GaN École thématique du CNRS, Montpellier

[6] Hilali A and Wazner A 1987 Formes super-irréductibles des systèmes différentiels linéaires Numer. Math. $50429-449$

[7] Jeannerod C P, Pflügel E 1999 A reduction algorithm for matrices depending on a parameter International Symposium on Symbolic and Algebraic Computation, Vancouver, Canada, ACM Press, July 1999, pp.121-128 
[8] Kane E O 1967 The kp method (Semiconductors and semimetals, Academic Press) p 75

[9] Kato T 1980 Perturbation theory for linear operators (Berlin: Springer)

[10] Löwdin P O 1951 A Note on the Quantum-Mechanical Perturbation Theory Journal of Chemical Physics 19 (11) 1396-1401

[11] Monagan M share/linalg/eigen/eigen.mws MaPLE computer algebra system

[12] Moro J, Burke J V and Overton M L 1997 On the Lidskii-Vishik-Lyusternik perturbation theory for eigenvalues of matrices with arbitrary Jordan structure SIAM J. Matrix Anal. Appl. 18 793-817

[13] Moser J 1960 The order of a singularity in Fuchs' theory Math. Z. 379-398

[14] Pflügel E 1998 Résolution symbolique des systèmes différentiels linéaires PhD Thesis, Université Joesph Fourier, Grenoble

[15] Van Hoeij M The IntBasis package MaPLE share library

[16] Wilkinson J H 1965 The algebraic eigenvalue problem (Oxford) 